[1] J. L. Hodeau, V. Favre-Nicolin, S. Bos, H. Renevier, E. Lorenzo, J. F. Berar, Chem. Rev.. 2001, 101, 1843.

[2] S. Welzmiller, P. Urban, F. Fahrnbauer, L. Erra, O. Oeckler, J. Appl. Crystallogr. 2013, 46, 769.

Keywords: thermoelectrics, resonant scattering, element distribution

\section{MS46-O2 Electronic depth profiles with atomic layer resolution from Resonant X-ray Reflectivity}

Jochen Geck ${ }^{1,2}$, M. Zwiebler ${ }^{1}$, B. Gray ${ }^{3}$, J. Chakhalian ${ }^{3}$, J. Freeland $^{4}$, F. He ${ }^{5}$, A. Koitzsch ${ }^{1}$, P. Komissinskiy ${ }^{6}$, E. Schierle ${ }^{7}$, S. Sutarto $^{5}$, U. Treske ${ }^{1}$, M. Vafaee ${ }^{6}$, E. Weschke ${ }^{7}$, G. A. Sawatzky ${ }^{8}$, L. Alff ${ }^{6}$, S. Macke ${ }^{9,10}$, J. E. Hamann-Borrero ${ }^{1}$

1. Leibniz Institute for Solid State and Materials Research, IFW-Dresden, Helmholtzstr. 20, 01069 Dresden, Germany

2. Institut für Strukturphysik, Technische Universität Dresden, D-01062 Dresden, Germany

3. Department of Physics, University of Arkansas, Fayetteville, Arkansas 70701, USA

4. Advanced Photon Source, Argonne National Laboratory, Argonne, Illinois 60439, USA

5. Canadian Light Source, University of Saskatchewan, Saskatoon, Saskatchewan, S7N0X4, Canada

6. Institute of Materials Science, Technische Universität Darmstadt, 64287 Darmstadt, Germany

7. Helmholtz-Zentrum Berlin für Materialien und Energie, Albert-Einstein-Str. 15, D-12489 Berlin, Germany

8. Department of Physics and Astronomy, University of British Columbia, Vancouver, V6T1Z1, Canada

9. Max Planck-UBC Centre for Quantum Materials, Vancouver, V6T1Z1, Canada

10. Max Planck Institute for Solid State Research, Heisenbergstr. 1, 70569 Stuttgart, Germany

email: J.Geck@ifw-dresden.de

Resonant $\mathrm{X}$-ray reflectivity (RXR) provides a unique experimental tool to study the surface and buried interfaces of oxide heterostructures. The analysis of reflectivity data usually assumes homogeneous properties throughout the volume of the constituent materials, i.e., the internal atomic structure of these materials is usually neglected. However, when the x-ray energy is tuned to an absorption edge, this approximation can cease to provide a good description of the experiment, because lattice planes with and without the elements at resonance will interact with the photons very differently. As a result, RXR can also provide important and very useful information about a heterostructure at the atomic level. We have therefore developed a scheme for analyzing RXR-data, which takes the atomic structure of a material into account by "slicing" it into atomic planes with characteristic optical properties. Using $\mathrm{LaSrMnO}_{4}$ and $\mathrm{YBa}_{2} \mathrm{Cu}_{3} \mathrm{O}_{7}$ as examples, we discuss the implications of this approach. Our analysis not only allows to determine structural information such as interface terminations and stacking of atomic layers, but also enables to extract depth-resolved spectroscopic information with atomic resolution, thus further enhancing the capability of the technique to study emergent phenomena at surfaces and interfaces.

Keywords: oxide heterostructures, resonant soft x-ray scattering 